\title{
Ultrasound assessment of the inferior vena cava for fluid responsiveness: easy, fun, but unlikely to be helpful
}

\author{
Scott J. Millington, MD
}

Received: 24 November 2018/Revised: 18 December 2018/Accepted: 19 December 2018/Published online: 27 March 2019

(C) Canadian Anesthesiologists' Society 2019

For medical disciplines responsible for the care of severely ill patients, arguably nothing is more desperately needed than a practical and accurate tool to predict fluid responsiveness (FR). Defined as the physiologic state where the administration of an intravenous fluid bolus will cause an increase in stroke volume $^{1}$, it is crucial to understand FR for four reasons:

1) Intravenous fluids are, appropriately, the mainstay of early resuscitation because of their availability, low cost, ease of administration, and potential to improve oxygen delivery;

2) After the very initial stages of resuscitation, critically ill patients consistently have a near 50\% probability of being in an FR state, ${ }^{2,3}$ indicating that clinicians are typically operating in a zone of perfect uncertainty;

3) Inadequate fluid administration is generally felt to be harmful ${ }^{4,5}$;

4) Overzealous fluid administration is associated with increased mortality. ${ }^{6,7}$

Intravenous fluid, just as with any drug, must be administered in exactly the right dose. Sepsis, as the most common cause of shock faced by most acute care providers, illustrates the clearest example. As the lessons of early antibiotic therapy and adequate source control have achieved the level of common wisdom, ${ }^{8}$ and as once promising adjunct therapies such as vasopressin, corticosteroids, and activated protein $\mathrm{C}$ have become mired in uncertainty (or worse), ${ }^{5}$ the physician presiding over a patient in septic shock is often left with one major decision-how much fluid should I give?

This article is accompanied by an editorial. Please see Can J Anesth 2019; 66: this issue.

S. J. Millington, MD ( $)$

Department of Intensive Care, The Ottawa Hospital, University of Ottawa, Box 207, 501 Smyth Road, Ottawa, ON K1H 8L6,

Canada

e-mail: smillington@toh.ca
We have tools that are easy to deploy. Performing a physical examination, assessing vital signs, or measuring central venous pressure all fall into this category, but none has any meaningful correlation to FR. ${ }^{9,10}$ We have tools that seem to accurately predict FR, such as passive leg raise ${ }^{11}$ or pulse pressure variability, ${ }^{12}$ but neither tool is easy. Passive leg raise requires that an estimate of stroke volume be used as the dependent variable,${ }^{11}$ committing the operator to a measurement device that is either very invasive (right heart catheterization), labor intensive and operator dependent (echocardiography), ${ }^{13}$ or of controversial precision (bioreactance ${ }^{14}$ or pulse contour analysis, ${ }^{15}$ among many others). Pulse pressure variability is not particularly invasive, but can only be reliably used for a small fraction of patients. ${ }^{16}$ Beyond being passively mechanically ventilated with large tidal volumes, patients must be in normal sinus rhythm and have relatively normal right ventricular function, pulmonary compliance, and pulmonary arterial pressures; patients who meet all six criteria are rare indeed.

Into this morass steps a tool billed as the solution to our important problem: assessment of the inferior vena cava (IVC) by ultrasound. Indeed, the last decade has seen an explosive increase in the use of this tool, and why not? With ultrasound machines residing in every nook and cranny of most every hospital, and with studies reaching back 15 years attesting to its accuracy, ${ }^{17,18}$ it seems at first glance to be the answer to our easiness/accurateness dilemma. Unfortunately, a more detailed examination of both the basic physiology and the base of evidence underpinning IVC analysis leads to the uncomfortable conclusion that it simply does not work as well as is generally perceived.

\section{Inferior vena cava analysis: the basics}

A detailed technical description of the ultrasound technique is beyond the scope of this paper and is 
well-described elsewhere. ${ }^{19}$ Suffice it to say that placing a phased-array transducer just beneath the patient's xiphoid process with the orientation marker directed cranially easily yields a view of the IVC in most patients. From here the operator can freeze the screen and measure the diameter of the IVC at end-expiration, where the intrathoracic pressure is closest to atmospheric pressure. A small IVC diameter is evidence of an FR state and a larger diameter the converse, although the appropriate cutoff point is difficult to pin down. Alternatively and more commonly, the variability of the IVC with respiration ( $\triangle \mathrm{IVC})$ is used as a marker of FR. Here, the size of the IVC at end-expiration is compared with that at end-inspiration, and a percentage change in size is calculated; M-mode is often used to simplify this task. The IVC will collapse on inspiration in patients breathing spontaneously and will distend for patients ventilated with positive pressure, but in either case the change in IVC diameter is the variable of interest. Larger values are taken as evidence of an FR state, but again an optimal threshold value is challenging to determine.

\section{Technical limitations}

There are several reasons to suspect that measurements, whether of IVC diameter or its variability with respiration, are likely inaccurate in many cases:

\section{Point of measurement}

The operator is typically advised to measure the IVC diameter $1-2 \mathrm{~cm}$ from the cavo-atrial junction, ${ }^{20}$ avoiding the hepatic vein. In a structure that averages $17 \mathrm{~mm}$ in the average adult ${ }^{21}$ and tends to flare as it approaches the right atrium, the chosen point of measurement can influence the result significantly.

Perpendicularity of measurement

Operators who favour M-mode for IVC measurement may fail to ensure that the IVC is perfectly perpendicular to the long axis of the vessel; such errors will cause the diameter to be overestimated.

Foreshortening error

Accurate measurement requires that the ultrasound plane transect the true middle of the vessel; measurements taken from other planes underestimate the true diameter. The IVC shifts roughly $4 \mathrm{~mm}$ in the medio-lateral plane with inspiration, ${ }^{22}$ introducing a significant measurement error.
Off-axis collapse

The IVC is typically measured in a sagittal plane, but when the IVC collapses (or expands) it does so in a plane that is not perfectly sagittal. ${ }^{22}$ If a sagittal plane is taken to be at $90^{\circ}$, the IVC typically collapses at about $115^{\circ}$, a further source of error in calculating $\triangle \mathrm{IVC}$.

Qualitative estimates

Busy clinicians are sometimes prone to looking at the IVC with ultrasound and making an "eyeball" estimate of whether its variability is sufficient to justify fluid administration. Such qualitative estimates are inaccurate, and can lead to erroneous conclusions in as many as one third of cases. ${ }^{23}$

Inter-rater reliability

The inter-rater reliability of IVC measurements is far from perfect (mean difference, 4\%; 95\% confidence interval [CI], $-30 \%$ to $38 \%$ in one study ${ }^{24}$; correlation coefficient, 0.6 ; $95 \% \mathrm{CI}, 0.45$ to 0.72 in another ${ }^{25}$ ) raising the uncomfortable possibility that two providers using the same tool at the same time could come to opposite conclusions for the same patient.

Confusing the aorta for the IVC

As parallel and similarly sized structures in direct proximity, the IVC and aorta can be mistaken for one another; the frequency of this error is not known.

\section{Confounding factors}

Even if accurately measured, there are several reasons to be concerned that the IVC may not relate to FR in the manner expected:

\section{Thoracic factors}

The change in size of the IVC depends entirely on swings in intrathoracic pressure. Therefore, in spontaneously breathing patients, the magnitude of the respiratory effort represents a crucial and impossible to quantify variable. It is easy to imagine a patient who is so dyspneic that they cause the collapse of a "full" IVC, and contrarily a patient who is barely breathing and therefore does not collapse an "empty" IVC.

The situation in mechanically ventilated patients is not much easier. While rendering a patient passive on the ventilator through use of paralytic agents or heavy sedation 
and raising tidal volumes to high levels (unfortunately, 8$10 \mathrm{~mL} \cdot \mathrm{kg}^{-1}$ in almost every study) is a well-meaning (though not benign) attempt to remove some of the ambiguity associated with variations in intrathoracic pressure, it does nothing to alleviate the variability associated with poor lung compliance, to name but one confounding factor.

\section{Cardiac factors}

Patients with right ventricular dysfunction typically have a chronically enlarged IVC, confounding attempts to interpret the size of the vessel.

\section{Abdominal factors}

Patients who are obese or who have an ileus present a formidable obstacle to accurate measurement of the IVC. Beyond that, elevated intra-abdominal pressure presents an underappreciated barrier where there appears to be a nearly complete loss of the relationship between IVC size and FR in patients with an intra-abdominal pressure of just 12 $\mathrm{mmHg}$ or more. ${ }^{26}$ This is a very common state for critically ill patients. ${ }^{27}$

\section{The evidence: spontaneously breathing patients}

While challenging to study because of variable patient populations, severities of illness, and research environments, there has been a good amount of scholarly work done in this specific area. For spontaneously breathing patients there have been nine studies, but two feature unusual patient populations (severely pre-eclamptic women ${ }^{28}$ and children in the neurosurgical operating room). ${ }^{29}$ Of the remaining seven, three studies were negative. ${ }^{30-32}$ Two others yielded borderline results, with the authors commenting that the "IVC cannot reliably predict FR" in one ${ }^{33}$ and "caval index does not reliably predict FR" in the other. ${ }^{34}$

The first positive result comes from a study requiring patients to perform a standardized and quantitative inspiratory effort, ${ }^{35}$ a technique that cannot be applied to patients who are dyspneic, supine, confused, or intubated, greatly limiting its applicability to critical care. The second ${ }^{36}$ is a relatively large $(n=124)$ emergency department study, which yielded a strong positive result but was criticized for the use of a somewhat controversial tool to estimate FR (thoracic bioreactance), the late enrolment of patients ( $16 \mathrm{hr}$ after presentation, having already received $4 \mathrm{~L}$ of fluid on average), and the low inter-rater reliability (0.67) between experts.
Given the above information, it seems fair to conclude that the literature does not clearly support the use of $\Delta \mathrm{IVC}$ to predict FR in spontaneously breathing patients. This fact, when combined with the worrisome technical and confounding factors as described in the introduction, is a strong argument in favour of abandoning the tool for this patient population until further data becomes available.

\section{The evidence: mechanically ventilated patients}

Inferior vena cava size

Starting with the most straightforward question, can simply measuring the size of the IVC at end-expiration predict FR in mechanically ventilated patients? Unfortunately, the answer to this question appears to be "no". In what is by far the largest and best study on this subject to date, Vieillard-Baron et al. $^{26}$ considered 540 consecutive critically ill patients. This cohort featured patients who were intubated and passive on the ventilator, in conditions ideal to study IVC analysis such as we currently understand them. When the end-expiratory IVC dimeter was measured, the IVC diameter distribution curves for the FR and non-FR groups overlapped so extensively as to remove any potential discriminatory value. The authors conclude that end-expiratory IVC diameter "poorly predicts FR because of a broad range of uncertainty".

The same study also approached this problem using a "grey zone" approach. ${ }^{37-39}$ If one were to require, at a minimum, a sensitivity and specificity of $80 \%$, one could set two thresholds for end-expiratory IVC diameter based on the data in this cohort of patients. An IVC diameter of $<$ $13 \mathrm{~mm}$ would predict FR with a specificity of $80 \%$, whereas an IVC diameter of $>25 \mathrm{~mm}$ would exclude an FR state with a sensitivity of $80 \%$. Translating this into plain English, a clinician would give fluids to a patient with an IVC diameter of $13 \mathrm{~mm}$ or less, withhold fluid from patients with an IVC diameter of $25 \mathrm{~mm}$ or more, and be unable to interpret any IVC diameter between 13 and 25 $\mathrm{mm}$. This seems like an excellent solution to the problem at hand, except that in this cohort, a full $71 \%$ of all patients fell within this grey zone, rendering the tool useless in a large majority of cases.

\section{Inferior vena cava variability}

There are eight studies examining the use of $\triangle \mathrm{IVC}$ for determining FR in mechanically ventilated patients. Of the eight, three are negative $e^{40-42}$ and the remaining five $\mathrm{e}^{17,18,43-45}$ are all small $(n=23-49)$ single centre efforts, which are further limited by variability in patient populations and illness severity. Such studies, while 
extremely valuable, should be repeated with much larger sample sizes prior to being accepted as generalizable and true. This is especially important when, as in this case, there is a mix of negative and positive results.

Fortunately, in the same large cohort of 540 critically ill patients described above, Vignon et al. ${ }^{2}$ also examined the utility of IVC variability in determining FR, and compared it with three other commonly used methods. Of the four methods tested, IVC variability performed worst of all, with an area under the receiver operator curve of only 0.635 .

Examining the Vignon et l. $^{2}$ data from a Bayesian perspective highlights how the analysis of IVC variability is unlikely to be useful at the bedside. Beginning with a pre-test probability for FR of $50 \%$, a very reasonable proposition for most patients early in their critical illness, ${ }^{3}$ a positive result on the test $(\Delta \mathrm{IVC}>8 \%)$ would only increase the post-test probability of an FR state to $65 \%$ (specificity 70\%, positive likelihood ratio 1.83). A negative result $(\Delta \mathrm{IVC}<8 \%)$ would decrease the post-test probability of an FR state to 39\% (sensitivity 55\%, negative likelihood ratio 0.64). This, of course, leaves the clinician precisely where they started, in a position of great uncertainty. Beginning the exercise with perfect uncertainty (a 50\% pre-test probability), after examining the IVC there is either a $65 \%$ chance of an FR state with a positive test or a $39 \%$ chance with a negative test. In both scenarios, far too much uncertainty remains to act confidently.

Perhaps then a grey zone analysis ${ }^{26}$ can improve the usability of IVC variability; the authors of the Vignon study ${ }^{2}$ are equally helpful here. Setting a threshold for IVC variability at $3 \%$ results in an optimal sensitivity (to rule out an FR state) of $74 \%$. Setting a threshold of $18 \%$ optimizes the specificity (to rule in an FR state) at $90 \%$. Translating this to plain English, a clinician would give fluids to a patient with a $\triangle \mathrm{IVC}$ of $18 \%$ or more, withhold fluid from patients with a $\triangle \mathrm{IVC}$ of $3 \%$ or less, and would be unable to interpret any $\triangle \mathrm{IVC}$ value that fell between $3 \%$ and $18 \%$. Again, this seems like a wonderful solution to this vexing problem; but, importantly, 53\% of patients in this study fell in the range between $3 \%$ and $18 \%$ (data obtained through personal correspondence with the author), rendering them uninterpretable and the test therefore unhelpful.

\section{Conclusions}

The use of ultrasound for resuscitative purposes is expanding rapidly, and there is excellent reason to suspect that it will be of benefit to patients. ${ }^{46}$ Further study is, of course, required, and an important part of the safe evolution of point-of-care ultrasound will be to recognize when certain aspects, like IVC analysis, are being applied overzealously.

There are multiple technical reasons to fear for the accuracy of IVC measurements. Even if we assume the measured value is true, there are a host of confounding factors, of which almost every patient will have at least one. For spontaneously breathing patients, the weight of currently available evidence suggests that the tool has poor predictive value, and thus supports abandoning the use of IVC analysis in this patient population until new data shifts this balance.

For mechanically ventilated patients, the story is slightly more complex. A series of small studies has yielded mixed results, but the best data to date suggests that there is no single threshold which can be used as a cutoff value to discriminate between patients who are FR and those who are not. A grey zone analysis improves the performance characteristics of the test, but most patients will fall within the grey zone where the results simply cannot be interpreted. Looming unhelpfully over this discussion is the additional fact that a minority of mechanically ventilated patients are likely to meet criteria for IVC analysis on any given day, analogous to the problem faced by pulse pressure variability. ${ }^{16}$ Put simply, the tool is rarely useful where real uncertainty exists.

There have been four recent meta-analyses on this topic. ${ }^{47-50}$ The two most recent conclude simply that "respiratory variation in IVC diameter has limited ability to predict FR" 46 and that "ultrasound evaluation of the diameter of the IVC and its respiratory variations does not seem to be a reliable method to predict FR" ${ }^{50}$

It is likely that IVC analysis will eventually be seen as a modern day central venous pressure; rapidly and enthusiastically adopted not because it was accurate but because it was easy, and because we were desperate for a solution to an important problem. We are still desperate for a practical solution to this same FR problem, but it seems clear that IVC analysis will not be helpful for a large majority of patients and should therefore be abandoned in most situations.

Conflicts of interest None declared.

Editorial responsibility This submission was handled by Dr. Hilary P. Grocott, Editor-in-Chief, Canadian Journal of Anesthesia.

Funding information Not funded.

\section{References}

1. Marik PE, Lemson J. Fluid responsiveness: an evolution of our understanding. Br J Anaesth 2014; 112: 617-20.

2. Vignon $P$, Repesse $X$, Begot $E$, et al. Comparison of echocardiographic indices used to predict fluid responsiveness 
in ventilated patients. Am J Respir Crit Care Med 2017; 195: 1022-32.

3. Michard F, Teboul JL. Predicting fluid responsiveness in ICU patients : a critical analysis of the evidence. Chest 2002; 121 : 2000-8.

4. Rivers E, Nguyen B, Havstad S, et al. Early goal-directed therapy in the treatment of severe sepsis and septic shock. N Engl J Med 2001; 345: 1368-77.

5. Rhodes A, Evans LE, Alhazzani W, Levy MM, Antonelli M. Surviving sepsis campaign: international guidelines for management of sepsis and septic shock: 2016. Intensive Care Med 2017; 43: 304-77.

6. Boyd JH, Forbes J, Nakada TA, Walley KR, Russell A. Fluid resuscitation in septic shock: a positive fluid balance and elevated central venous pressure are associated with increased mortality. Crit Care Med 2011; 39: 259-65.

7. Alsous F, Khamiees M, DeGirolamo A, Amoateng-Adjepong Y, Manthous $C A$. Negative fluid balance predicts survival in patients with septic shock: a retrospective pilot study. Chest 2000; 117 : 1749-54.

8. Gao F, Melody T, Daniels DF, Giles S, Fox $S$. The impact of compliance with 6-hour and 24-hour sepsis bundles on hospital mortality in patients with severe sepsis: a prospective observational study. Crit Care 2005; 9: R764-70.

9. Bentzer P, Griesdale D, Boyd J, MacLean K, Sirounis D, Ayas $N T$. Will this hemodynamically unstable patient respond to a bolus of intravenous fluids? JAMA 2016; 316: 1298-309.

10. Marik PE, Cavallazzi $R$. Does central venous pressure predict fluid responsiveness? An updated meta-analysis and a plea for some common sense. Crit Care Med 2013; 41: 1774-81.

11. Cavallaro F, Sandroni $C$, Marano $C$, et al. Diagnostic accuracy of passive leg raising for prediction of fluid responsiveness in adults: systematic review and meta-analysis of clinical studies. Intensive Care Med 2010; 36: 1475-83.

12. Marik PE, Cavallazzi R, Vasu T, Hirani A. Dynamic changes in arterial waveform derived variables and fluid responsiveness in mechanically ventilated patients: a systematic review of the literature. Crit Care Med 2009; 37: 2642-7.

13. Wetterslev M, Mфller-Sфrensen H, Johansen RR, Perner A. Systematic review of cardiac output measurements by echocardiography vs. thermodilution: the techniques are not interchangeable. Intensive Care Med 2016; 42: 1223-33.

14. Kupersztych-Hagege E, Teboul JL, Artigas A, et al. Bioreactance is not reliable for estimating cardiac output and the effects of passive leg raising in critically ill patients. Br J Anaesth 2013; 111: 961-6.

15. Saraceni E, Rossi S, Persona P, et al. Comparison of two methods for cardiac output measurement in critically ill patients. Br J Anaesth 2011; 106: 690-4.

16. Mahjoub $Y$, Lejeune $V$, Muller L. Evaluation of pulse pressure variation validity criteria in critically ill patients: a prospective observational multicentre point-prevalence study. $\mathrm{Br} \mathrm{J}$ Anaesth 2014; 11: 681-5.

17. Barbier C, Loubieres $Y$, Schmit $C$, et al. Respiratory changes in inferior vena cava diameter are helpful in predicting fluid responsiveness in ventilated septic patients. Intensive Care Med 2004; 30: 1740-6.

18. Feissel M, Michard F, Faller JP, Teboul JL. The respiratory variation in inferior vena cava diameter as a guide to fluid therapy. Intensive Care Med 2004; 30: 1834-7.

19. Denault AY, Langevin S, Lessard MR, Courval JF, Desjardins G. Transthoracic echocardiographic evaluation of the heart and great vessels. Can J Anesth 2018; 65: 449-72.

20. Lang RM, Badano IP, Mor-Avi V. Recommendations for cardiac chamber quantification by echocardiography in adults: an update from the American Society of Echocardiography and the
European Association of Cardiovascular Imaging. Eur Heart $\mathbf{J}$ Cardiovasc Imaging 2015; 16: 233-70.

21. Mintz GS, Kotler MN, Parry WR, Iskandrian AS, Kane SA. Realtime inferior vena caval ultrasonography: normal and abnormal findings and its use in assessing right heart function. Circulation 1981; 64: 1018-25.

22. Blehar DJ, Resop D, Chin B, Dayno M, Gaspari R. Inferior vena cava displacement during respirophasic ultrasound imaging. Crit Ultrasound J 2012; 4: 18.

23. Duwat A, Zogheib E, Guinot PG, et al. The gray zone of the qualitative assessment of respiratory changes in inferior vena cava diameter in ICU patients. Crit Care 2014; 18: R14.

24. Bowra J, Uwagboe V, Goudie A, Reid C, Gillett M. Interrater agreement between expert and novice in measuring inferior vena cava diameter and collapsibility index. Emerg Med Australas 2015; 27: 295-9.

25. Akkaya A, Yesilaras M, Aksay E, Sever M, Atilla OD. The interrater reliability of ultrasound imaging of the inferior vena cava performed by emergency residents. Am J Emerg Med 2013; 31: 1509-13.

26. Vieillard-Baron A, Evrard B, Repesse X, et al. Limited value of end-expiratory inferior vena cava diameter to predict fluid responsiveness impact of intra-abdominal pressure. Intensive Care Med 2018; 44: 197-203.

27. Malbrain M, Chiumello D, Pelosi $P$, et al. Prevalence of intraabdominal hypertension in critically ill patients: a multicentre epidemiological study. Intensive Care Med 2004; 30: 822-9.

28. Brun C, Zieleskiewicz L, Textoris $J$, et al. Prediction of fluid responsiveness in severe preeclamptic patients with oliguria. Intensive Care Med 2013; 39: 593-600.

29. Byon HJ, Lim CW, Lee JH, et al. Prediction of fluid responsiveness in mechanically ventilated children undergoing neurosurgery. Br J Anaesth 2013; 110: 586-91.

30. Williams $K$, Ablordeppey $E$, Theodoro $D$, et al. The diagnostic accuracy of inferior vena cava collapsibility versus passive leg raise testing in determining volume responsiveness in emergency department patients with shock. Proceedings of the 40th Critical Care Congress, Society of Critical Care Congress. Crit Care Med 2011; 39: 8 .

31. Corl K, Napoli AM, Gardiner F. Bedside sonographic measurement of the inferior vena cava caval index is a poor predictor of fluid responsiveness in emergency department patients. Emerg Med Australas 2012; 24: 534-9.

32. Airapetian A, Maizel J, Alyamani $O$, et al. Does inferior vena cava respiratory variability predict fluid responsiveness in critically ill patients? Crit Care 2015; 19: 400.

33. Muller L, Bobbia X, Toumi M, et al. Respiratory variations of inferior vena cava diameter to predict fluid responsiveness in spontaneously breathing patients with acute circulatory failure: need for a cautious use. Crit Care 2012; 16: R188.

34. de Valk S, Olgers TJ, Holman M, Ismael F, Ligtenberg JJ, Ter Maaten $J C$. The caval index: a adequate non-invasive ultrasound parameter to predict fluid responsiveness in the emergency department? BMC Anesthesiol 2014; 14: 114.

35. Preau S, Bortolotti P, Colling D, et al. Diagnostic accuracy of the inferior vena cava collapsibility to predict fluid responsiveness in spontaneously breathing patients with sepsis and acute circulatory failure. Crit Care Med 2017; 45: e290-7.

36. Corl KA, George NR, Romanoff $J$, et al. Inferior vena cava collapsibility detects fluid responsiveness among spontaneously breathing critically-ill patients. J Crit Care 2017; 41: 130-7.

37. Vos JJ, Poterman M, Salm PP, et al. Noninvasive pulse pressure variation and stroke volume variation to predict fluid responsiveness at multiple thresholds: a prospective observational study. Can J Anesth 2015; 62: 1153-60. 
38. Cannesson M. The, "grey zone" or how to avoid the binary constraint of decision-making. Can J Anesth 2015; 62: 1139-42.

39. Coste J, Pouchot J. A grey zone for quantitative diagnostic and screening tests. Int J Epidemiol 2003; 32: 304-13.

40. Sobczyk D, Nycz K, Andruszkiewicz P, Wierzbicki K, Stapor M. Ultrasonographic caval indices do not significantly contribute to predicting fluid responsiveness immediately after coronary artery bypass grafting when compared to passive leg raising. Cardiovasc Ultrasound 2016; 14: 23.

41. Charbonneau H, Riu B, Faron M, et al. Predicting preload responsiveness using simultaneous recordings of inferior and superior vena cavae diameters. Crit Care 2014; 18: 473.

42. Theerawit $P$, Morasert $T$, Sutherasan $Y$. Inferior vena cava diameter variation compared with pulse pressure variation as predictors of fluid responsiveness in patients with sepsis. J Crit Care 2016; 36: 246-51.

43. Moretti R, Pizzi B. Inferior vena cava distensibility as a predictor of fluid responsiveness in patients with subarachnoid hemorrhage. Neurocrit Care 2010; 13: 3-9.

44. Machare-Delgado E, Decaro M, Marik PE. Inferior vena cava variation compared to pulse contour analysis as predictors of fluid responsiveness: a prospective cohort study. J Intensive Care Med 2011; 26: 116-24.

45. Lu N, Xi X, Jiang L, Yang D, Yin K. Exploring the best predictors of fluid responsiveness in patients with septic shock. Am J Emerg Med 2017; 35: 1258-61.
46. Millington SJ. Cardiac ultrasound is a competency of critical care medicine. Crit Care Med 2017; 45: 1555-7.

47. Zhang Z, XuX, Ye S, Xu L. Ultrasonographic measurement of the respiratory variation in the inferior vena cava diameter is predictive of fluid responsiveness in critically ill patients: systematic review and meta-analysis. Ultrasound Med Biol 2014; 40: 845-53.

48. Huang $H$, Shen $Q$, Liu $Y, X u H$, Fang $Y$. Value of variation index of inferior vena cava diameter in predicting fluid responsiveness in patients with circulatory shock receiving mechanical ventilation: a systematic review and meta-analysis. Crit Care 2018; 22: 204.

49. Long E, Oakley E, Duke T, Babl FE; Paediatric Research in Emergency Departments International Collaborative (PREDICT). Does respiratory variation in inferior vena cava diameter predict fluid responsiveness: a systematic review and meta-analysis. Shock 2017; 47: 550-9.

50. Orso D, Paoli I, Piani T, Cilenti FL, Cristiani L, Guglielmo N. Accuracy of ultrasonographic measurements of inferior vena cava to determine fluid responsiveness: a systematic review and metaanalysis. J Intensive Care Med 2018; DOI: https://doi.org/10. $1177 / 0885066617752308$.

Publisher's Note Springer Nature remains neutral with regard to jurisdictional claims in published maps and institutional affiliations. 